\title{
Observation of fluctuation modulation of tunnel junctions by applied ac stress in carbon polyvinylchloride composites
}

\author{
E. K. Sichel, ${ }^{*}$ Ping Sheng, ${ }^{\dagger}$ J. I. Gittleman, and S. Bozowski \\ RCA Laboratories, Princeton, New Jersey 08540
}

(Received 31 March 1981)

\begin{abstract}
Modulation of small tunneling barriers by thermally activated voltage fluctuations is observed in the composite material carbon polyvinylchloride consisting of large conducting carbon chains and aggregates separated by narrow insulating gaps. The effect is experimentally determined by applying an ac stress to the sample and measuring the change in resistivity per unit strain as a function of temperature. After determining the tunneling parameters $T_{0}$ and $T_{1}$ from the temperature dependence of the resistivity, the temperature dependence of the change in resistivity per unit strain is calculated based on the fluctuation-induced tunneling theory in which the tunneling barrier is lowered and narrowed by thermally activated voltage fluctuations. Good agreement is obtained between the experimental results and the fluctuation-induced tunneling theory.
\end{abstract}

We have studied the effect of modulation of tunnel junction width on the conductivity of a composite material consisting of large conducting chains and aggregates separated by narrow insulating gaps. Carbon polyvinylchloride (C-PVC) consists of graphitic carbon spheres, $100-400 \AA$ in diameter, embedded in a PVC-based resin. The carbon spheres cluster into filaments and aggregates $\sim 1$ $\mu \mathrm{m}$ in size randomly oriented with small gaps of PVC between aggregates. ${ }^{1}$ The conductivity of the C-PVC has been shown to be due to fluctuationinduced tunneling through the gaps between carbon aggregates. $^{2,3}$ In this paper we present evidence that due to their small dimensions the tunneling barriers in the C-PVC composites are modified by thermally activated voltage fluctuations across the gaps. The effect is demonstrated by pressure modulation of the tunneling gap.

The temperature dependence of the resistivity of C-PVC composites can be expressed ${ }^{2}$ as

$$
\rho=\rho_{0} \exp \left(\frac{T_{1}}{T+T_{0}}\right),
$$

where $T_{1}$ is a measure of the energy required to move an electron across the insulating gap and $T_{0}$ is the temperature much below which the resistivity reduces to temperature-independent tunneling. Temperature fluctuations generate voltages $V \sim(k T / C)^{1 / 2}$ across the insulating gaps of capaci- ty $C$ which lower and narrow the potential barrier. In the case of small junction capacity, the thermal fluctuations give rise to large voltage fluctuations. For the samples studied in this work, the values $w \sim 75 \AA$, junction area $A \sim 380 \AA^{2}$, and the height of the potential barrier $V_{0} \sim 0.092 \mathrm{eV}$ are consistent with the values of $T_{0}$ and $T_{1}$ found to fit Eq. (1). The values of $T_{1}$ and $T_{0}$ depend on the tunneling parameters but cannot by themselves give information about the dependence of the tunneling on any particular parameter. We have designed an experiment which can determine the dependence of the tunneling on the single parameter $w$, the junction gap width, and thus make detailed comparisons with the tunneling theory.

The junction width $w$ was modulated by applying an ac mechanical stress at $25 \mathrm{~Hz}$ to the CPVC. The sample was mounted in a stainless steel yoke with a piezoelectric transducer, and the yoke was tightened until the stress applied to the sample and transducer produced a static strain of about $0.5 \%$, measured with a strain gauge glued to the sample, a cube of about $7 \mathrm{~mm}$ on a side. A small dc bias current was passed through the sample, and the dc voltage developed across potential leads on the sample face was used to measure the resistance; the ac voltage developed when the transducer was driven at $25 \mathrm{~Hz}$ was used to measure the piezoresistance. Similarly, the dc voltage in the strain gauge measured gauge resistance and therefore the 
static strain; the ac voltage across the strain gauge was used to measure the rms strain on top of the static strain.

At each temperature $R_{\mathrm{dc}}$ and $(\Delta R)_{\mathrm{ac}}$ were measured for both the sample and strain gauge resistances with a four-lead method. The small static strain was not sufficient to alter measurably the distribution ${ }^{4}$ of junction widths as measured by $T_{1}$ and $T_{0}$. This can be seen in Fig. 1 where $\rho(T)$ curves are shown for a sample with and without static stress.

The transducer was energized to provide an alternating strain $\Delta l / l$ of $1.1-1.3 \times 10^{-6}$ along the axis of the dc current flow and the static strain. The sample resistance and the strain gauge resistance were thus modulated at the driving frequency and the ac components of the sample and strain gauge voltages were detected with a lock-in amplifier (PAR 124). The entire yoke assembly was immersed in a helium bath for temperatures less than 4.2 $\mathrm{K}$ and suspended in helium vapor for tempera-

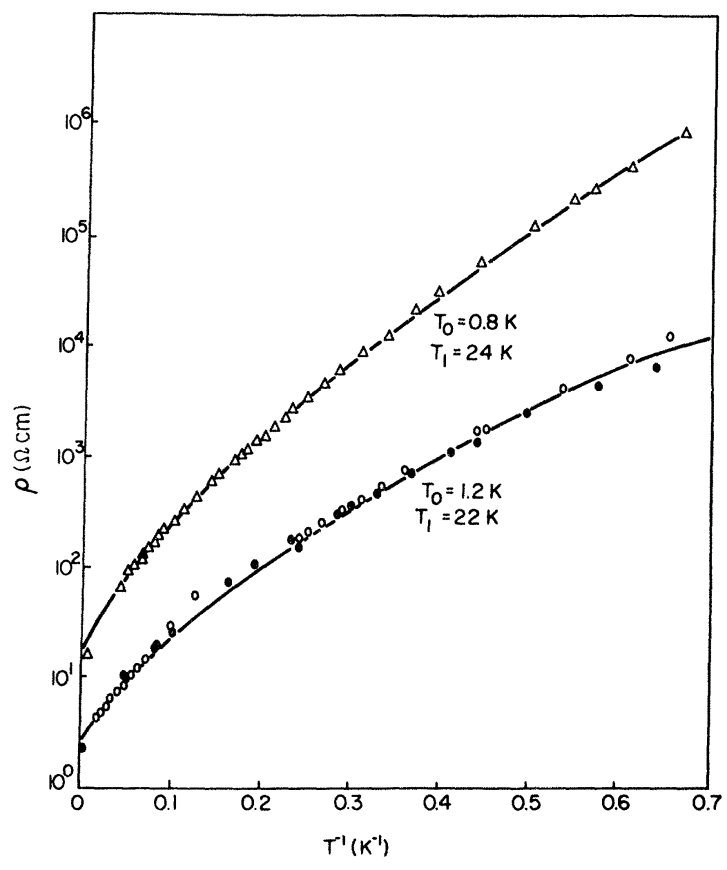

FIG. 1. Electrical resistivity as a function of $1 / T$ for 15 -wt. \% type- $A$ carbon black (open circles) and 35wt. \% type- $B$ carbon black (triangles) in PVC-based resin with no applied stress. Also shown is the difference between $\rho$ of type- $A$ carbon black with (solid circles) and without (open circles) a static strain of $0.5 \%$. The difference is so small it can barely be seen on the scale of the figure. The variation of $T_{0}$ and $T_{1}$ under stress is within the confidence limits of $T_{0}$ and $T_{1}$. Similar results were obtained for type- $B$ carbon black. tures above $4.2 \mathrm{~K}$. Care was taken to assure that the samples were Ohmic at all currents and temperatures and to assure negligible joule heating.

The samples used in this study were 15 -wt. \% type- $A$ carbon black and $35-$ wt. $\%$ type- $B$ carbon black. Type- $A$ carbon black is a carbon composed of hollow spheres approximately $350 \AA$ in diameter with $10-15$ - $\AA$-thick walls. Type- $B$ carbon black is a solid carbon sphere $180 \AA$ in diameter. It is interesting that the parameters $T_{0}$ and $T_{1}$ for the two carbon composites are almost the same (see Fig. 1). It is not surprising that the weight percent loadings of the two samples (15\% and $35 \%)$ are different, while $T_{0}$ and $T_{1}$ are similar because it has been shown by Coutts ${ }^{5}$ that PVC penetrates the hollow type $A$ carbon black. The effective volume percent of PVC outside the spheres is similar for the two carbon samples studied here.

The ac strain is a measure of the junction width; the rms change in resistivity per unit rms strain $(\Delta S \equiv \Delta l / l)$ was measured as a function of temperature. It has been shown ${ }^{4}$ that

$\frac{1}{\rho} \frac{d \rho}{d S}=\frac{1}{\rho_{0}} \frac{d \rho_{0}}{d S}+\frac{1}{T+T_{0}} \frac{d T_{1}}{d S}-\frac{T_{1}}{\left(T+T_{0}\right)^{2}} \frac{d T_{0}}{d S}$,

where $\left(1 / \rho_{0}\right)\left(d \rho_{0} / d S\right)$ can be regarded as a constant. $T_{1}$ and $T_{0}$ are functions of tunnel junction parameters. In order to calculate $d T_{1} / d S$ and $d T_{0} / d S$, it is necessary to examine the change in the tunneling barrier with respect to the variation of the junction width $w$. The potential barrier we consider is the image-force-corrected rectangular barrier $^{6} V(u)$ :

$$
V(u)=V_{0}\left(1-\frac{\lambda}{u(1-u)}\right)
$$

where $V_{0}$ is the height of the rectangular barrier in the absence of image-force correction, $u=x / w, x$ being the spatial coordinate in the tunneling direction, and

$$
\lambda=\frac{0.795 e^{2}}{4 w K V_{0}},
$$

where $e$ is the electronic charge and $K$ is the dielectric constant of the insulating barrier. In Eq. (3) the zero of the potential is defined as the Fermi level of the conducting particles. It can be shown ${ }^{4}$ that the parabolic barrier, from which Eq. (1) is derived, ${ }^{2}$ is an excellent approximation to $V(u)$ with $\lambda \cong 0.07$. As a result, almost identical temperature dependences can be obtained by consider- 
ing either the parabolic potential barrier or the image-force-corrected barrier with $\lambda \cong 0.07$. However, because in the parabolic barrier approximation the barrier shape is rigidly fixed regardless of the junction width, $V(u)$ would be a more realistic basis for the calculation of $d T_{1} / d S$ and $d T_{0} / d S$.

$V(u)$ is a peaked function of $u$. In the presence of an electric field, the maximum of $V(u), V_{m}$, would decrease in magnitude. If we denote $\epsilon_{0}$ as the field strength at which $V_{m}=0$, then the parameters $T_{1}$ and $T_{0}$ are defined ${ }^{4}$ as

$$
\begin{aligned}
& T_{1}=a \epsilon_{0}^{2} / k, \\
& T_{0}=T_{1} / 2 \chi w \xi_{0},
\end{aligned}
$$

where $a=w A / 8 \pi, A$ being the area of the junction, $\chi=\left(2 m V_{0} / \hbar^{2}\right)^{1 / 2}, k$ is Boltzmann's constant, and

$$
\xi_{0}=\int_{u_{1}}^{u_{2}}\left[\frac{V(u)}{V_{0}}\right]^{1 / 2} d u,
$$

where $u_{1}$ and $u_{2}\left(>u_{1}\right)$ are the two roots of $V(u)=0 . \epsilon_{0}$ can be expressed in the form $\epsilon_{0}=\epsilon_{v} \bar{\epsilon}_{0}$, where $\epsilon_{v}=V_{0} / e w$, and $\bar{\epsilon}_{0}$ is a dimensionless quantity which depends only on $\lambda$. Since $d S=d w / w$, we get

$$
\begin{aligned}
\frac{d T_{1}}{d S} & =w \frac{d}{d w}\left[\frac{A V_{0}^{2}}{8 \pi k e^{2}} \frac{1}{w} \bar{\epsilon}_{0}^{2}\right]=\left[2 \frac{w}{\bar{\epsilon}_{0}} \frac{d \lambda}{d w} \frac{d \bar{\epsilon}_{0}}{d \lambda}-1\right) T_{1} \\
& =-\left(2 \frac{\lambda}{\bar{\epsilon}_{0}} \frac{d \bar{\epsilon}_{0}}{d \lambda}+1\right) T_{1},
\end{aligned}
$$$$
\frac{d T_{0}}{d S}=\left(\frac{\lambda}{\xi_{0}} \frac{d \xi_{0}}{d \lambda}-2 \frac{\lambda}{\bar{\epsilon}_{0}} \frac{d \bar{\epsilon}_{0}}{d \lambda}-2\right) T_{0}
$$

Numerical calculation shows that

$$
\frac{\lambda}{\bar{\epsilon}_{0}} \frac{d \bar{\epsilon}_{0}}{d \lambda}=-1.24, \frac{\lambda}{\xi_{0}} \frac{d \xi_{0}}{d \lambda}=-0.4605
$$

for $\lambda=0.07$. Substituting these values into Eqs. (7) and (2) yields

$$
\frac{1}{\rho} \frac{d \rho}{d S}=\mathrm{const}+\frac{1.48 T_{1}}{T+T_{0}}-\frac{0.020 T_{1} T_{0}}{\left(T+T_{0}\right)^{2}} .
$$

From Eq. (8) it is seen that besides the additive constant, the temperature dependence of $(1 / \rho)(d \rho / d S)$ is completely determined once the values of $T_{0}$ and $T_{1}$ are fixed from the $\rho$ versus $T$ data.

In Fig. 2 the results for two C-PVC samples of different carbon type and weight percent of carbon are shown. The smooth curve is the fit to Eq. (8) using the values of $T_{0}$ and $T_{1}$ determined from Fig. 1 and treating the additive constant as an adjustable parameter. The values of $T_{0}$ and $T_{1}$ are almost the same for the two different carbon types and, as predicted by Eq. (8), the values of $(1 / \rho)(d \rho / d S)$ versus $T$ for the two carbons differ only by a constant value. In view of the good agreement between theory and the experimental results, the decreasing trend of $(1 / \rho)(d \rho / d S)$ has a simple physical interpretation. It is easy to verify that if the conduction were by simple tunneling, $\rho \sim \exp (B \chi w)$ and $(1 / \rho)(d \rho / d S)=B \chi w, B$ being some numerical constant. The product $\chi w$ is dependent only on the height, shape, and width of the tunneling barrier so the present observed decrease of $(1 / \rho)(d \rho / d S)$ as $T$ increases can be regarded as a decrease of $\chi w$ due to the effective lowering and narrowing of the barrier by thermally activated voltage fluctuations.

In conclusion, we have demonstrated that the

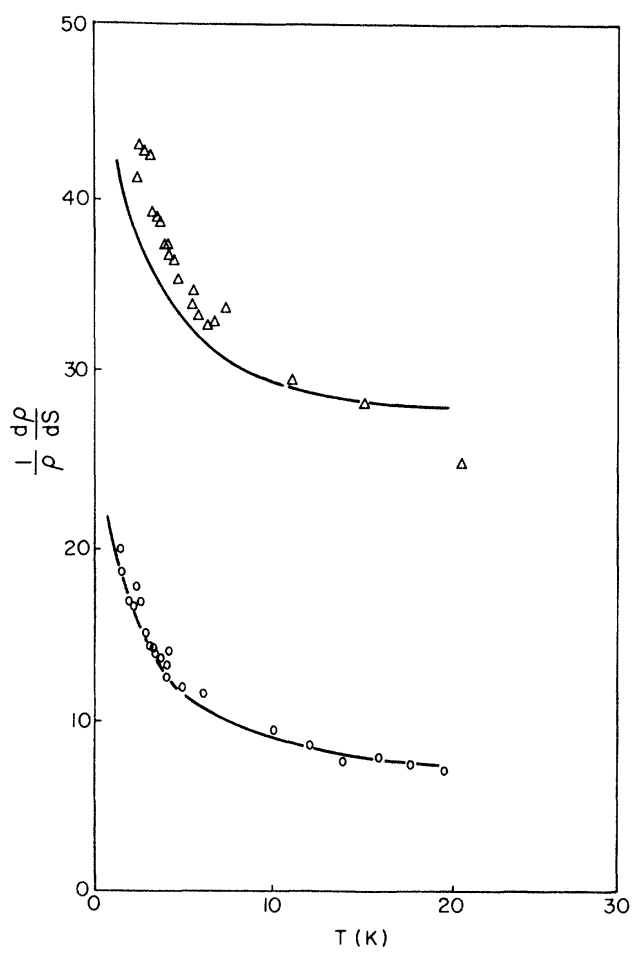

FIG. 2. $(1 / \rho)(d \rho / d S)$ as a function of temperature for 15 -wt. $\%$ of type- $A$ carbon black (circles) with a static strain of $0.5 \%$ and for $35-w t . \%$ type- $B$ carbon black (triangles) under a static stress of about $0.3 \%$. 
conductivity of chains and aggregates of carbon embedded in a PVC-based resin is limited by tunneling across minute gaps of small capacity. The tunnel barrier is lowered and narrowed by thermally activated voltage fluctuations. In this experi- ment we have singled out the tunneling parameter $w$ and obtained direct experimental evidence that the dependence of the conductivity on gap width is a function of temperature as predicted by the fluctuation-induced tunneling model.
"Present address: GTE Laboratories, 40 Sylvan Rd., Waltham, MA 02254.

Present address: Theoretical Sciences Group, Corporate Research Science Laboratories, Exxon Research and Engineering Co., P. O. Box 45, Linden, NJ 07036.

${ }^{1}$ M. Coutts (unpublished). Micrographs of C-PVC may also be found in the article by L. P. Fox, RCA Rev. 39, 116 (1978). Micrographs of carbon blacks may be found in Carbon Black, Jean-Baptiste Donnet and Andries Voet (Dekker, New York, 1976).

2Ping Sheng, E. K. Sichel, and J. I. Gittleman, Phys.
Rev. Lett. 40, 1197 (1978).

${ }^{3}$ E. K. Sichel, J. I. Gittleman, and Ping Sheng, Phys. Rev. B 18, 5712 (1978).

${ }^{4}$ The theory developed in Ref. 3 is based on single junction parameters. An extension of the theory to a distribution of junction sizes has been developed by Ping Sheng, Phys. Rev. B 21, 2180 (1980). The general conclusions of Ref. 3 are unchanged by considering a distribution of junction widths.

${ }^{5} \mathrm{M}$. D. Coutts (private communication).

6J. G. Simmons, J. Appl. Phys. 34, 1793 (1963). 